\title{
Recuperación de pérdida ósea en pseudoartrosis infectada de tibia con técnica de transporte óseo de De Bastiani
}

\author{
Bone loss recovery in tibia infected pseudoarthrosis with \\ De Bastiani bone transportation technique \\ Yessica Paola Alba Gutiérrez, * Jorge Enrique Cervantes Gudiño \\ *Médico residente de 4to año de Traumatología y Ortopedia; ${ }^{\ddagger}$ Médico adscrito a la Clínica de Ortopedia Pediátrica. \\ Centenario Hospital Miguel Hidalgo. Aguascalientes, México.
}

\begin{abstract}
Resumen
El transporte óseo se refiere a la capacidad de llevar hueso sano de un segmento diafisario hacia una zona de defecto óseo por cualquier causa, generando una osteogénesis paulatina y progresiva para cubrir un defecto. Objetivo: Reportar la función de la extremidad pélvica de dos casos de pacientes con pseudoartrosis infectada de tibia tratados con transporte óseo con método de fijador externo tipo monorriel en el Centenario Hospital «Miguel Hidalgo». Material y métodos: Se trata de un estudio retrospectivo, descriptivo y analítico. Se hizo una búsqueda en la base de datos del hospital donde se reportaron dos casos de pacientes con diagnóstico de pseudoartrosis infectada de tibia tratados con transporte óseo. Se recabaron las variables de estudio en una ficha predefinida incluyendo variables sociodemográficas. Resultados: Uno fue hombre y una mujer con edades de 15 y 17 años. Uno de los casos fue por fractura expuesta y una cerrada. La pérdida ósea promedio fue de $10 \mathrm{~cm}$. La fase de reposo fue de 15 días promedio. De acuerdo con los criterios de Paley, los resultados óseos fueron excelentes en un caso y bueno en el otro. En uno de los casos ya se retiró el fijador tipo monorriel y ya tiene consolidación completa. Los reactantes de fase aguda en los dos casos ya se encuentran negativos. Los resultados funcionales son excelentes en un paciente y bueno en el otro. Los dos pacientes cuentan ya con apoyo completo de la extremidad. Conclusión: Los resultados muestran que la transportación ósea mediante la técnica de De Bastiani es una buena alternativa para el manejo de pacientes con pérdida ósea mayor de tibia.
\end{abstract}

Palabras clave: Transporte óseo, pérdida ósea, tibia, fémur, pseudoartrosis.

\section{Abstract}

Bone transport refers to the ability to carry healthy bone from a diaphyseal segment to an area of bone defect due to any cause, generating a gradual and progressive osteogenesis to cover a defect. Objective: To report the function of the pelvic limb of two cases of patients with infected tibial nonunion treated with bone transport with the monorail-type external fixator method at the Centenario Hospital Miguel Hidalgo. Material and methods: This is a retrospective, descriptive and analytical study. A search was made in the hospital database where two cases of patients with a diagnosis of infected pseudoarthrosis of the tibia treated with bone transport were reported. The study variables were collected in a predefined file including sociodemographic variables. Results: One was male and one female aged 15 and 17 years. One of the cases was due to an open fracture and one was closed. Average bone loss was $10 \mathrm{~cm}$. The rest phase was an average of 15 days. According to Paley's criteria, bone results were excellent in one case and good in the other. In one of the cases, the monorail-type fixator was already removed and the patient is fully consolidated. The acute phase reactants in both cases are already negative. Functional results are excellent in one patient and good in the other. The two patients already have full limb support. Conclusion: The results show that bone transportations using the De Bastiani technique is a good alternative for the management of patients with major bone loss in the tibia.

Keywords: Bone transport, bone loss, tibia, femur, pseudoarthrosis.

Correspondencia:

Dra. Yessica Paola Alba Gutiérrez

E-mail: alba.gutierrez08@ hotmail.com

Citar como: Alba GYP, Cervantes GJE. Recuperación de pérdida ósea en pseudoartrosis infectada de tibia con técnica de transporte óseo de De Recibido: 03-04-2021. Aceptado: 13-04-2021. Bastiani. Orthotips. 2021; 17 (2): 75-81. https://dx.doi.org/10.35366/99900 


\section{Introducción}

Los defectos óseos pueden ser resultado de resecciones o diafisectomías por trauma, seudoartrosis con desvitalización ósea, infección, tumor o reemplazo protésico. ${ }^{1}$ El problema de las pérdidas óseas de tibia mayores de $3 \mathrm{~cm}$ es el difícil manejo que representan, ya que se tiene que recuperar una distancia considerable de hueso, que en ocasiones con los métodos convencionales (injertos óseos, estimulación eléctrica) y aun con los más sofisticados (injertos de peroné vascularizado, transportación ósea) suelen tener resultados catastróficos que pueden ser desde un proceso infeccioso agregado, deformidades residuales importantes, pérdidas del injerto óseo, hasta el tratamiento radical, ya que se encuentra comprometida la viabilidad de la extremidad. ${ }^{3}$

Se han descrito diferentes tratamientos para las pérdidas óseas mayores de $3 \mathrm{~cm}$ en las que se necesita recuperar la longitud del hueso, entre éstas: 1) desbridamiento y aplicación de injerto óseo autólogo, en éste se requiere revisar la viabilidad del injerto de manera progresiva, ya que gran parte sufre necrosis y pérdida del mismo; 2) trasplante de peroné vascularizado que ha probado ser una técnica efectiva, ya que no tiene que existir sustitución celular, sino que se integra consolidando en sus extremos como una fractura; este procedimiento requiere de al menos dos equipos quirúrgicos trabajando simultáneamente, uno en el sitio donador y otro en el área receptora, y de un cirujano con entrenamiento en cirugía vascular, la cirugía dura en promedio de 8 a 12 horas en manos expertas; 3 ) transporte óseo que permite la restitu- ción de la longitud requerida, así como la corrección de deformidades, procedimiento en el que una vez controlado el proceso infeccioso (si lo hay) sólo requiere de un equipo quirúrgico y dura en promedio dos horas la colocación del elongador y la realización de la corticotomía. ${ }^{4}$

llizarov en la década de los 50 acuñó el término osteogénesis por distracción (distracción del callo óseo) para describir la formación de hueso entre dos superficies óseas que eran separadas gradualmente, utilizando un aro modular como fijador externo y clavillos transfictivos unidos a los aros bajo tensión para estabilizar los fragmentos óseos. Este descubrimiento salvó extremidades, que de otro modo hubieran terminado en amputación, y el eventual regreso a diferentes niveles de actividad de pacientes deshabilitados. ${ }^{8}$ De Bastiani, por otra parte, empleó un fijador monolateral rígido no transfictivo con la capacidad de dinamización, utilizando tornillos troncocónicos, de colocación más sencilla y estable que daña menos a los tejidos blandos, además es más cómodo para el paciente. En la actualidad se siguen dos técnicas de transportación ósea: la de llizarov y la de De Bastiani. ${ }^{2}$

El presente estudio tiene como propósito reportar la función de la extremidad pélvica de tres casos de pacientes con pseudoartrosis infectada tratados con transporte óseo con método de fijador externo tipo monorriel en el Centenario Hospital Miguel Hidalgo.

\section{Material y métodos}

Se realizó un estudio retrospectivo, analítico y descriptivo de pacientes con pérdida ósea de miem-

Figura 1:

Fractura en agudo de

los pacientes.
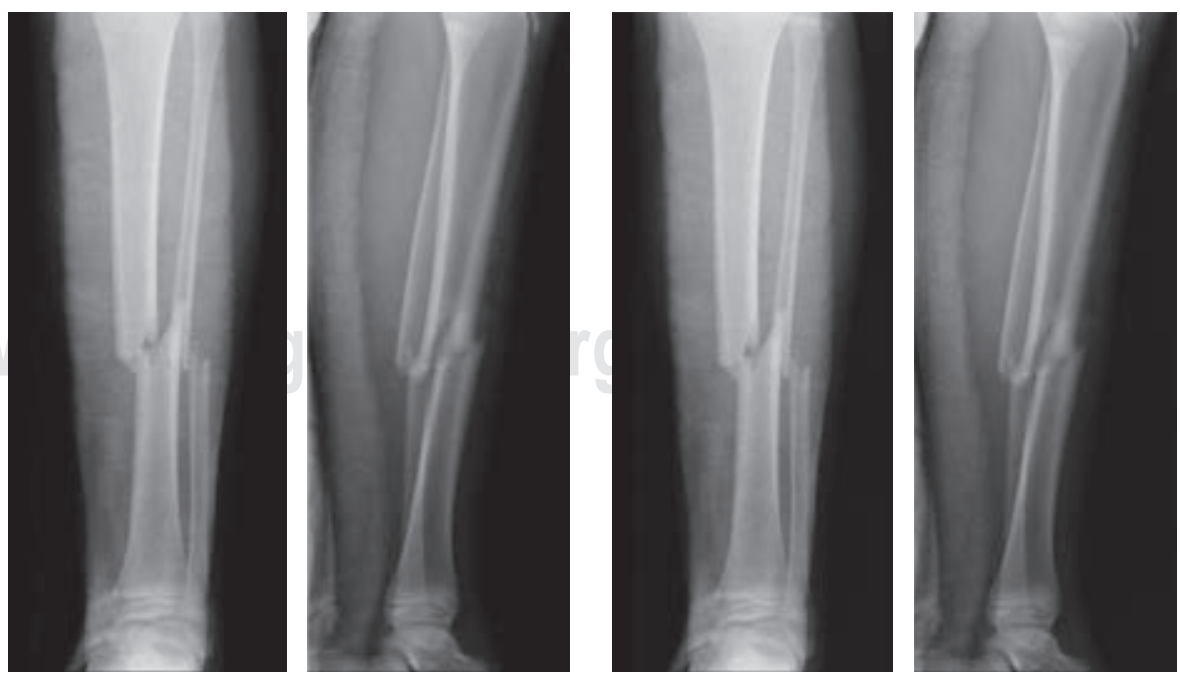

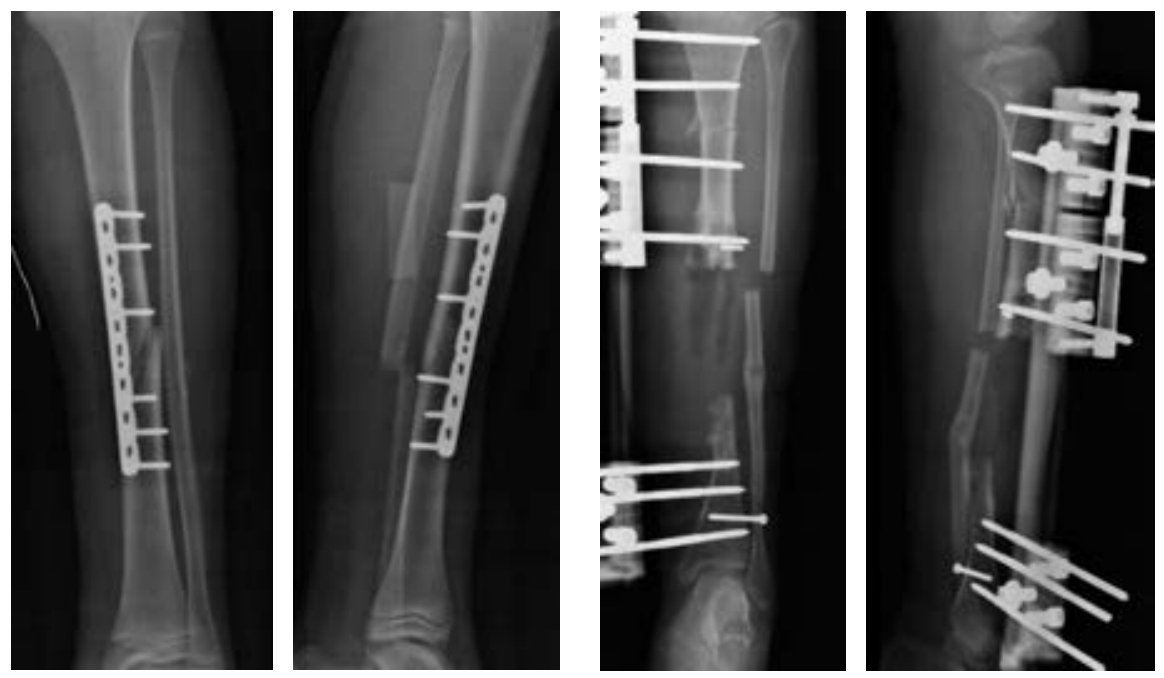

Figura 2:

Fijación primaria con placa LCP 4.5.

bros inferiores con pseudoartrosis infectada, que fueron tratados mediante transportación ósea con la técnica de De Bastiani (Tabla 1), con el sistema de fijador externo tipo monorriel. Se incluyeron en el estudio aquellos pacientes con pseudoartrosis infectada, de uno u otro sexo, con una pérdida ósea de tibia de más de $5 \mathrm{~cm}$, manejados en el servicio del Centenario Hospital «Miguel Hidalgo» (Figura 1). Se excluyeron los pacientes que no presentaron infección de pseudoartrosis. Se realizó una planeación quirúrgica para todos los casos mediante un protocolo preoperatorio general que incluyó estudios de laboratorio preoperatorios completos con reactantes de fase aguda, y se solicitaron controles de radiografías en la consulta externa. Sólo una paciente no comenzó el tratamiento inicial en este hospital, por lo que no contamos con las radiografías iniciales (Figura 2). Los resultados después de la corticalización del segmento transportado fueron evaluados desde el punto de vista óseo y funcional, utilizando el sistema descrito por Paley y colaboradores. Para los resultados óseos se valoraron cuatro criterios: consolidación, infección, deformidad y discrepancia de extremidades. ${ }^{9}$ Los resultados funcionales se basaron en cinco criterios: cojera significativa, deformidad rígida en equino del tobillo, distrofia de tejidos blandos (manifestada por hipersensibilidad dérmica o insensibilidad), dolor e inactividad (que el paciente quedara inhabilitado para las actividades diarias). De acuerdo con este sistema, un excelente resultado funcional es el que logra actividad sin ninguno de los otros criterios; un resultado bueno es el que realiza actividad pero con uno o dos de los otros cuatro criterios; regular es cuando logra actividad pero presenta tres o cuatro de los otros criterios o ameritó amputación; y malo en aquel que no logra actividad individual a pesar de los otros criterios. ${ }^{5}$

\section{Técnica quirúrgica}

El método de De Bastiani consiste en la estabilización de los fragmentos comprometidos con tornillos troncocónicos no transfictivos, los cuales se fijan a un riel rígido con tres cabezales deslizantes (sistema de fijación externa tipo monorriel). ${ }^{2}$ Con el paciente en decúbito dorsal bajo bloqueo subaracnoideo en mesa radiolúcida y con intensificador de imágenes, se determinan líneas paralelas a la superficie articular, se colocan tres cabezales provisionales en regleta de elongador, se coloca un primer tornillo en cabezal proximal paralelo a articulación de rodilla, el segundo tornillo en cabezal distal paralelo a articulación de tobillo y un tercer tornillo en cabezal intermedio paralelo a la diáfisis de la tibia, después se colocan los tornillos restantes, quedando tres tornillos por cabezal, se cambian cabezales provisionales por los definitivos (Figura 3). Después se realiza incisión de 1 a $1.5 \mathrm{~cm}$ en la región proximal o distal según el sitio seleccionado para la corticotomía hasta periostio, se incide éste de manera longitudinal y se procede a perforar agujeros en las caras anteromedial y anterolateral del hueso para perforar la cortical posterior con broca $3.2 \mathrm{~mm}$, mismos que se realizan con la menor distancia entre sí. A continuación, los agujeros se unen con un osteótomo 
una vez completada la osteotomía, porque la parte posterior se rompe de manera espontánea debido a la tensión aplicada antes del montaje. Se separan los segmentos mediante el distractor para asegurar que la osteotomía es completa y se confirma con el intensificador de imágenes. Se vuelven a aproximar los segmentos a distraer, sometiéndolos a una compresión leve y se aprietan las tuercas de bloqueo. Se cierra el periostio y la herida sin dejar drenajes. Se entrena al paciente y familiares sobre cómo realizar la distracción, cuándo iniciarla, frecuencia y sentido de la vuelta de la unidad de compresión-distracción, se le recomienda al paciente anotar en un cuaderno hora y fecha de distracción y sus observaciones como sensación de tracción, dolor o cualquier eventualidad. De no existir contraindicación alguna, se egresa al segundo día del postoperatorio. La fase de reposo dura en promedio 10 días. La distracción se realiza a razón de $1 \mathrm{~mm}$ por día, girando cuatro veces al día un cuarto de vuelta en sentido antihorario la unidad de compresión-distracción (0.25 $\mathrm{mm}$ cada 6 horas). Este promedio de distracción se acelera temporalmente cuando se observa una osificación rápida, o se reduce si la osificación es lenta o el paciente padece dolor o contractura muscular. La distracción es vigilada mediante controles radiográficos. Se cita a los 10 días del postoperatorio para valorar las heridas, retirar los puntos y verificar que entendió cómo iniciar la distracción. Tres citas semanales al inicio y quincenales posteriormente con radiografías de control. La fase de reposo dura en promedio un día. La distracción se realiza a razón de $1 \mathrm{~mm}$ por día, girando cuatro veces al día un cuarto de vuelta en sentido antihorario la unidad de compresión-distracción ( $0.25 \mathrm{~mm}$ cada 6 horas). Este promedio de distracción se acelera temporalmente cuando se observa una osificación rápida, o se reduce si la osificación es lenta o el paciente padece dolor o contractura muscular. La distracción es vigilada mediante controles radiográficos. Se cita a los 10 días del postoperatorio para valorar las heridas, retirar los puntos y verificar que entendió cómo iniciar la distracción. Nueva cita a los 10 días con radiografía de control para valorar que la distracción se realiza de manera correcta. Se le recomienda la carga parcial de aproximadamente $15 \mathrm{~kg}$. Después se cita cada 30-40 días con radiografías de control para comprobar que se produzca la osteogénesis,

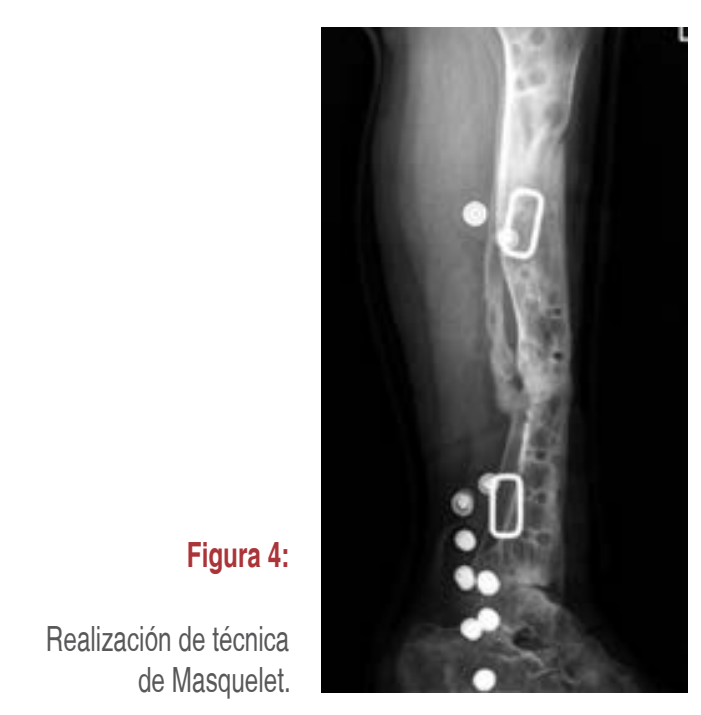

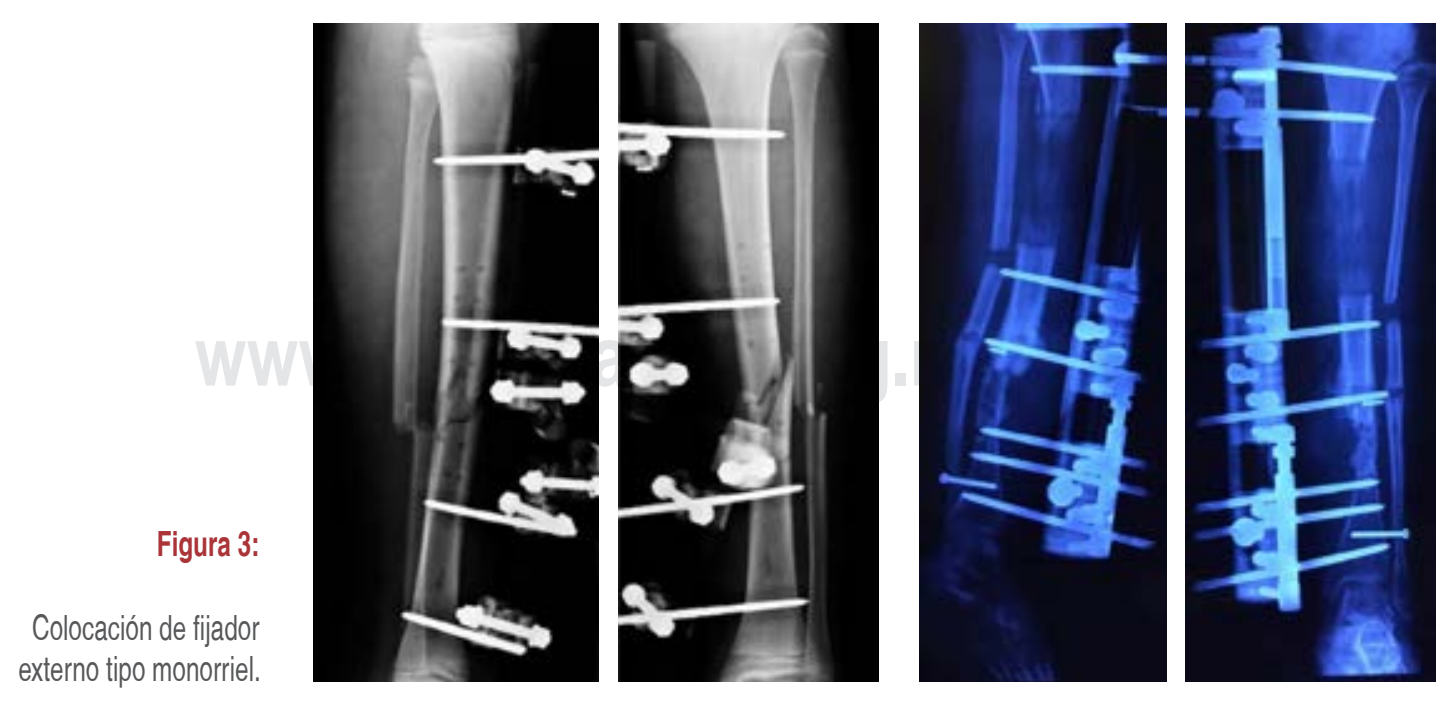




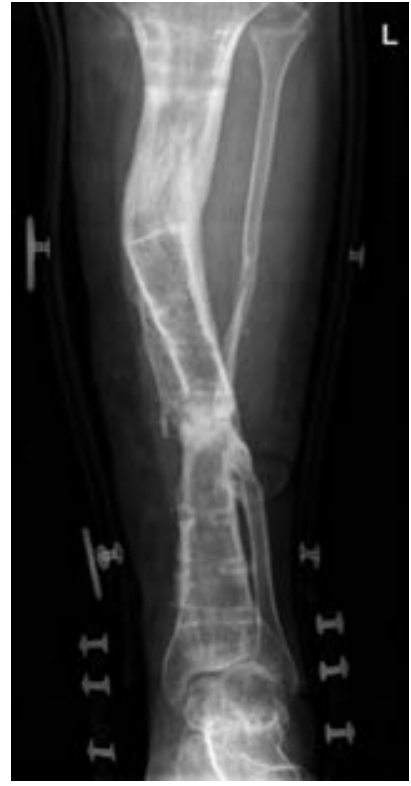

Figura 5:

Retiro de fijador externo, se observa la consolidación y recuperación ósea.

en cuyo caso se continúa la transportación. Si la densidad del neoformado es pobre pero uniforme, se suspende la distracción por una o dos semanas. Si el neoformado es irregular, se comprime el segmento uno o dos centímetros a la misma velocidad de la distracción hasta que el callo sea uniforme, a continuación se reinicia la transportación. Al final de la transportación, cuando hay contacto de los dos fragmentos, la radiografía debe mostrar un callo uniforme (Figura 4). Se bloquea el cabezal del alargador para mantener el hueso nuevo en neutralización estable, el compresor-distractor es entonces retirado. Cuando así se requiere, una nueva cirugía

puede realizarse para reavivar los bordes de la zona de contacto y colocar injerto para acortar el tiempo de consolidación, el soporte de carga en esta fase de neutralización se incrementa gradualmente en función del grado de mineralización del neoformado. Cuando la radiografía demuestra que el segmento es uniformemente denso y opaco, se dinamiza el elongador aflojando la tuerca del cabezal proximal y se bloquean las del cabezal medio y distal. Una vez que se ha logrado la corticalización completa, se procede al retiro del elongador y los tornillos troncocónicos, se realiza en quirófano y bajo anestesia general endovenosa (Figura 5).

\section{Resultados}

De acuerdo con los criterios de selección, fueron incluidos un total de dos pacientes con pérdida ósea de tibia y fémur por pseudoartrosis infectada que recibieron tratamiento mediante transportación ósea con la técnica de De Bastiani durante el periodo de estudio. Uno fue del sexo masculino (50\%) y uno femenino (50\%). La edad promedio fue de 16 años (rango de 15 a 17 años). Una de las pérdidas óseas (50\%) se presentaron como secuelas de fracturas expuestas y una de fracturas cerradas (50\%). La fractura expuesta fue en la tibia izquierda y la cerrada en la tibia izquierda. Una de las fracturas se reportó por causa espontánea, idiopática, la fractura expuesta por caída de una caja de camioneta. En la fractura expuesta, el tratamiento inicial consistió en desbridamiento y fijación externa, con reducción abierta, fijación interna con placa de compresión bloqueable (LCP) 3.5. Para la fractura
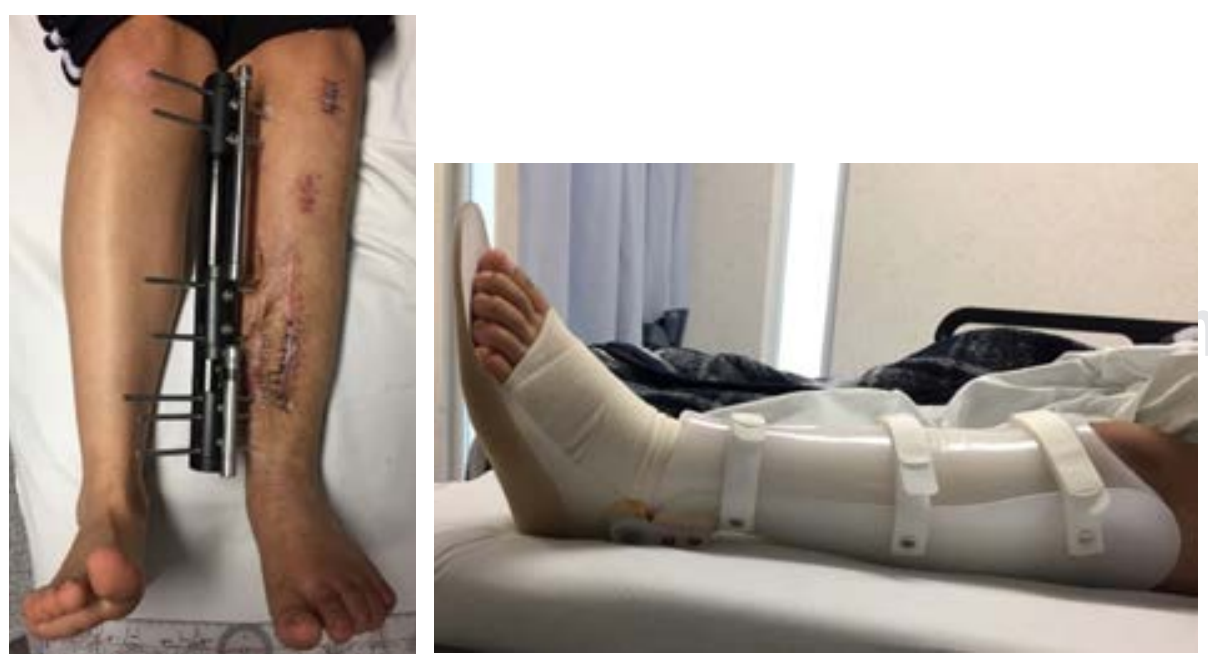

Figura 6:

Imágenes actuales de los pacientes. 
Tabla 1: Características de pacientes.

\begin{tabular}{|c|c|c|c|c|c|c|c|c|c|}
\hline Caso & & Hueso & Sexo & Edad & Lado & $\begin{array}{l}\text { Tipo de } \\
\text { fractura }\end{array}$ & Causa & Tx inicial & Pérdida $(\mathrm{cm})$ \\
\hline 1 & 2018-06176 & Tibia & Femenino & 17 & Izquierdo & Cerrada & Espontánea & Placa LCP 3.5 & 10 \\
\hline 2 & 2009-15954 & Tibia & Masculino & 15 & Izquierdo & $\begin{array}{l}\text { Expuesta } \\
\text { grado I }\end{array}$ & $\begin{array}{l}\text { Caída de caja } \\
\text { de camioneta }\end{array}$ & Placa LCP 3.5 & 13 \\
\hline
\end{tabular}

Tabla 2: Resultados funcionales.

\begin{tabular}{|c|c|c|c|c|c|c|}
\hline Caso & Claudicación & Equino & $\begin{array}{l}\text { Distrofia de tejidos } \\
\text { blandos }\end{array}$ & Dolor & Actividad & $\begin{array}{l}\text { Resultado funcional } \\
\left(\text { (Paley }^{*}\right)\end{array}$ \\
\hline 1 & Sí & Sí & Sí & No & Limitada & Bueno \\
\hline 2 & No & No & Sí & No & Normal & Bueno \\
\hline
\end{tabular}

* Sistema de clasificación de Paley y cols.

cerrada de tibia en reducción abierta fijación interna con placa LCP 3.5. Sólo un paciente no comenzó su tratamiento quirúrgico en este hospital (fractura cerrada de tibia) llegado a la consulta externa con placa fatigada y en pseudoartrosis infectada. La longitud de pérdida ósea promedio fue de $10 \mathrm{~cm}$ (rango de 7 a $11 \mathrm{~cm}$ ). El sitio de la corticotomía en los dos casos fue diafisario proximal. La transportación ósea se inició en promedio con los 15 días después de la corticotomía (rango de 10 a 15 días). A los dos pacientes se les realizó técnica Masquelet durante el procedimiento. La velocidad de distracción promedio fue de $0.85 \mathrm{~mm} /$ día (rango de 0.57 a $0.98 \mathrm{~mm} /$ día). El tiempo de consolidación y corticalización del segmento transportado fue de 14.5 meses (rango de 6 a 22 meses), sólo en un caso fue de tres años (el caso de la fractura expuesta). El índice de corticalización promedio fue de $1.9 \mathrm{~cm} / \mathrm{mes}$ (rango de 0.8 a $3.2 \mathrm{~cm} / \mathrm{mes}$ ). Los pacientes lograron la consolidación del segmento transportado aunque uno de los casos aún continúa con el fijador monorriel. Dos pacientes presentaron deformidad residual del segmento transportado, uno con retroverso de $6^{\circ} \mathrm{y}$ valgo de 50; el segundo presentó anteverso de $15^{\circ}$. Discrepancia de las extremidades inferiores ocurrió en los dos pacientes, de 1.5, $2 \mathrm{~cm}$ y $2 \mathrm{~cm}$. De acuerdo con el sistema de clasificación de Paley, dos pacientes tuvieron resultados óseos excelentes $(71 \%)$ y uno buenos (29\%) (Tabla 2).

Desde el punto de vista funcional, uno cuenta por el momento con ortesis tipo Sarmiento y uno con apoyo de muletas. Ninguno cuenta con gonalgia residual. Un paciente presentó deformidad en equino del tobillo y un paciente evolucionó con marcha claudicante importante. Basado en los criterios de Paley, un resultado funcional fue excelente (50\%) y uno bueno $(50 \%)$. La complicación encontrada durante la transportación ósea fue la infección (Tabla 3). Se estuvieron tratando con el Servicio de Infectología basándose en cultivos en los cuales los últimos se reportaron sin desarrollo, y sólo uno con $S$. epidermidis y con reactantes de fase aguda negativos en los dos casos actualmente. Un paciente (33.3\%) refirió dolor durante la transportación, el cual cedió con analgésicos orales. Al interrogar a cada uno de los pacientes acerca de la satisfacción del tratamiento, a pesar del tiempo prolongado y las complicaciones presentadas durante la transportación, los dos respondieron estar satisfechos.

\section{Discusión}

Tal y como se realizó en el presente estudio, el inicio de la transportación ósea posterior a la corticotomía se recomienda entre los primeros siete a 15 días, ya que si se inicia en forma previa el neoformado no es de calidad adecuada y se fractura; después de 15 días la corticotomía consolida y es difícil iniciar la transportación. La velocidad de distracción recomendada es de $1 / 4$ vuelta al día, y el índice de corticalización reportado en la bibliografía es de $1 \mathrm{~cm} / \mathrm{mes}$ en niños y de 2 a $3 \mathrm{~cm} / \mathrm{mes}$ en adultos. Los resultados óseos y funcionales obtenidos en nuestro estudio son muy similares a 
Tabla 3: Reporte de estudios infecciosos.

\begin{tabular}{cccccc}
\hline Caso & $\begin{array}{c}\text { Fecha de último } \\
\text { cultivo }\end{array}$ & Resultado & $\begin{array}{c}\text { Fecha } \\
\text { LABS }\end{array}$ & VSG & PCR \\
\hline 1 & $28 / 12 / 2018$ & Sin desarrollo & $22 / 01 / 2019$ & 16 & 5 \\
2 & $12 / 03 / 2018$ & S. epidermidis & $13 / 03 / 2018$ & 40 & 2.94 \\
\hline
\end{tabular}

LABS = Fecha de la toma de la VSG y PCR; VSG = Velocidad de sedimentación globular; $\mathrm{PCR}=$ Reacción en cadena de la polimerasa

los reportados por Paley y Tucker, quienes realizan este procedimiento. Una de las principales complicaciones encontradas durante la transportación ósea fue el proceso infeccioso, el cual estuvo siendo valorado por el Servicio de Infectología con antibioticoterapia basada en los cultivos. El dolor durante la transportación ósea es una complicación esperada secundaria a la distracción de los tejidos blandos, en esta evaluación, un paciente refirió dolor leve, el cual cedía a analgésicos orales; cuando la sintomatología no cede, es posible detener la distracción por un breve periodo, rehabilitar movilizando las articulaciones próximas y reiniciar posteriormente la distracción. Ningún caso presentó fractura durante el proceso; la fractura del neoformado es una de las complicaciones que pueden presentarse, según lo reportado por Aronson y Tucker. ${ }^{6}$ De acuerdo con nuestros resultados, consideramos que la transportación ósea es una buena alternativa en el manejo de las pérdidas óseas mayores de tibia, por lo que recomendamos su uso en casos de pérdidas importantes. ${ }^{7}$ Sin embargo, debe considerarse que durante el seguimiento pueden presentarse algunas complicaciones o dificultades técnicas, por lo que este procedimiento debe realizarse por cirujanos con un entrenamiento adecuado (Figura 6).

\section{Conclusiones}

La técnica de De Bastiani es una técnica reproducible y con buenos resultados, según los obtenidos en nuestro estudio para nuestro medio.

\section{Referencias}

1. Cierny G 3rd, Zorn KE. Segmental tibial defects. Comparing conventional and Ilizarov methodologies. Clin Orthop Relat Res. 1994; (301): 118-123.

2. Orona-Ontiveros JJ, Vázquez-Talavera M. Recuperación de pérdidas óseas de tibia mediante transporte óseo con fijadores externos. Acta Ortop Mex. 2005; 19 (2): 42-48.

3. Marti GJC, López de Arriba GF. Transporte óseo. Rev Esp Cir Osteoart. 1992; 27: 277-282.

4. Olivera Laborda HF, Viacava Siazaro FA, Noria A, Rey Nande R. Tratamiento de defectos masivos de tibia. Técnica de transporte óseo con fijador AO. Anfamed. 2018; 5 (2): 130-140.

5. Aktuglu K, Erol K, Vahabi A. Ilizarov bone transport and treatment of critical-sized tibial bone defects: a narrative review. J Orthop Traumatol. 2019; 20 (1): 22.

6. Li R, Zhu G, Chen C, Chen Y, Ren G. Bone transport for treatment of traumatic composite tibial bone and soft tissue defects: any specific needs besides the llizarov technique? Biomed Res Int. 2020; 2020: 2716547.

7. Kinik H, Kalem M. llizarov segmental bone transport of infected tibial nonunions requiring extensive debridement with an average distraction length of 9,5 centimetres. Is it safe? Injury. 2019: S0020-1383(19)30818-6.

8. Mora-Ríos FG, Antonio-Romero CE, Mejía-Rohenes LC, Cortés-Gómez J, López-Marmolejo A, Franco-López EA. Seudoartrosis infectada en tibia, un caso infantil. Caso clínico y revisión de la literatura. Acta Ortop Mex. 2012; 26 (4): 255-259.

9. Jovanovic V, Vukasinovic Z, Seslija I, Zivkovic Z. Treatment of septic pseudoarthrosis of tibia by llizarov method: case report. Srp Arh Celok Lek. 2010; 138 (9-10): 643-646.

\section{Conflicto de intereses}

Este estudio no cuenta con ningún conflicto de intereses. 